\title{
Eine diskursanalytische Untersuchung zur Volkszählung von 1890 am Beispiel des Aussiger Anzeigers und der Zeitung Česká stráž
}

\section{Discourse Analytical Inquiries into the 1890 Census, Based on the Aussiger Anzeiger and Česká stráž}

Tereza Hrabcová

\begin{abstract}
Considering the increasingly turbulent coexistence of the German-speaking population and the Czech-speaking population of the Bohemian lands in the $19^{\text {th }}$ century, it seems useful to explore when the notions "nationality" and "nation" started to spread in the public. When searching for the answer to this question, particularly daily as well as weekly newspapers are helpful because they were the dominant mass media in the $19^{\text {th }}$ as well as at the beginning of the $20^{\text {th }}$ century. Thus, on the one hand newspapers reflected the public discourse, on the other hand they were also shaping it to a large extent. This paper therefore analyses the public discourse on the 1890 census in one German-language newspaper and one Czech-language newspaper from Bohemia, in order to see, whether or to what extent the categories "nationality" and "nation" had already been present in public discourse. The comparative approach is essential in order to show the discourse from a more complex perspective. In the paper, the main attention is being paid to the analysis of argumentation.
\end{abstract}

\section{Keywords}

discourse analysis, 1890 census, Aussiger Anzeiger, Česká stráž, national political discourse, journalistic texts, Bohemian Lands 


\section{Einleitung}

In Anbetracht des turbulenten Zusammenlebens und allmählichen „Auseinanderlebens “1 der deutschsprachigen und der tschechischsprachigen Bevölkerung der böhmischen Länder in der zweiten Hälfte des 19. und ersten Hälfte des 20. Jahrhunderts scheint es sinnvoll, der Frage nachzugehen, wann die Konzepte „Nation“ und „Nationalität“ in der breiteren Öffentlichkeit Fuß zu fassen begannen. Bei der Suche nach der Antwort lohnt sich ein genauerer Blick auf die Texte in Tages- und Wochenzeitungen, da gerade sie als die dominanten Massenmedien ihrer Zeit den öffentlichen Diskurs teils widerspiegelten, teils mitprägten. Das Thema der Volkszählung von 1890 ist für eine solche diskursanalytische Untersuchung insofern geeignet, als bei dieser Volkszählung bereits zum zweiten Mal die Umgangssprache der Bewohner Österreich-Ungarns mit erfasst wurde ${ }^{2}$ und daher in der Presse verfolgt werden kann, ob, eventuell inwiefern die Umgangssprache zu nationalen bzw. nationalistischen Zwecken instrumentalisiert wurde. Von besonderer Bedeutung ist daher vor allem die Analyse der Argumentation, die einen wichtigen Platz im diskurslinguistischen Instrumentarium einnimmt.

Für diese Fallstudie wurde ein deutschsprachiges und ein tschechischsprachiges Periodikum gewählt, wobei der Fokus der Arbeit auf der deutschsprachigen Zeitung liegt. Gerade aufgrund einer solchen konfrontativen Analyse kann ein möglichst plastisches Bild des Diskurses skizziert werden.

,Diskursanalyse“ wird in dieser Arbeit in Anlehnung an Ingo Warnke und Jürgen Spitzmüller als eine Disziplin verstanden, die ihren Schwerpunkt ,jenseits textueller Grenzen“3 hat, sich also nicht auf Einzeltexte konzentriert, sondern auf ein „Ensemble von Texten“, 4 in denen ein bestimmtes Thema präsent ist. „In erster Linie ist Diskursanalyse [...] ein Verfahren, um die Behandlung bestimmter Themen in einer Gesellschaft zu ermitteln. "5 Das Textkorpus ist dabei nicht mit dem Diskurs gleichzusetzen; ein Diskurs ist aber „nur über Textkorpora zugänglich“6. Mithilfe von Textkorpora können „Teilmengen von Diskursen für die linguistische Analyse verfügbar [...] [ge]mach[t] "7 werden. Von Belang für diskursanalytische Untersuchungen ist außerdem die Annahme von der

1 HOENSCH, Jörg K.: Geschichte Böhmens. Von der slavischen Landnahme bis zur Gegenwart. München 1997, S. 357.

2 Vgl. TEIBENBACHER, Peter, KRAMER, Diether, GÖDERLE, Wolfgang: An Inventory of Austrian Census Materials, 1857-1910. Final Report. In: Mosaic Working Paper WP2012-007, Max Planck Institute for Demographic Research: December 2012. http://www.censusmosaic.org/sites/default/files/downloads/publications/mosaicWP/MOSAIC-WP-2012-007.pdf (17. 5. 2016).

3 WARNKE, Ingo, SPITZMÜLLER, Jürgen: Methoden und Methodologie der Diskurslinguistik - Grundlagen und Verfahren einer Sprachwissenschaft jenseits textueller Grenzen. In: WARNKE, Ingo, SPITZMÜLLER, Jürgen (Hrsg.): Methoden der Diskurslinguistik. Sprachwissenschaftliche Zugänge zur transtextuellen Ebene. Berlin, New York 2008, S. 3-54.

4 NIEHR, Thomas: Einführung in die linguistische Diskursanalyse. Darmstadt 2014, S. 29.

5 NIEHR [Anm. 4], S. 127.

$6 \quad$ NIEHR [Anm. 4], S. 32.

7 NIEHR [Anm. 4], S. 33. 
„sprachlichen Konstruktion der Wirklichkeit“": Menschen erschließen die Wirklichkeit allem voran durch Sprache, und insofern kommt sprachlichen Äußerungen - gerade auch denjenigen in den Zeitungen oder in anderen Massenmedien - in Denkprozessen, Erkenntnis- und Deutungsprozessen eine besondere Rolle zu. Wichtig ist dabei auch, dass „[d]er Verbreitungsgrad massenmedialer Botschaften [...] sehr hoch [ist], die Chance, Zugang zu den Massenmedien zu erhalten, dagegen vergleichsweise gering. ${ }^{\text {"9 }}$ Dies wird in der vorliegenden Untersuchung ebenfalls exemplifiziert.

\section{Analysekorpus und diskursiver Kontext}

Der deutschsprachige Aussiger Anzeiger wurde im August 1857 in der nordböhmischen Stadt Aussig [Ústí nad Labem] ins Leben gerufen, ${ }^{10}$ die tschechischsprachige Zeitung Česká stráž [Tschechische Wacht] erschien zum ersten Mal am 1. Januar 1890 in der Landeshauptstadt Prag, fand aber ebenfalls in Nordböhmen Verbreitung. Während der Aussiger Anzeiger als ein zweimal wöchentlich erscheinendes Periodikum gegründet wurde, zeitweise jedoch nur einmal wöchentlich erschien, im Oktober 1902 aber schließlich zur täglichen Erscheinungsweise wechselte und in Aussiger Tagblatt umbenannt wurde, kam die tschechischsprachige Zeitung Česká stráž zumeist nur einmal pro Woche heraus.

Als Stichtag für die Volkszählung galt der 31. Dezember 1890, sodass in der vorliegenden Analyse sowohl alle relevanten Texte des Jahrgangs 1890 berücksichtigt wurden als auch alle Texte von 1891, welche rückblickend den Verlauf der Volkszählung und ihre Ergebnisse thematisierten.

Das Jahr 1890 stand außerdem im Zeichen der sogenannten Wiener Punktationen, eines Ausgleichsversuchs zwischen dem deutschen und dem tschechischen Bevölkerungsteil der böhmischen Länder. Offiziell hatten die Ausgleichsgespräche am 4. Januar 1890 begonnen und nahmen zunächst einen ziemlich raschen Verlauf: Bereits am 19. Januar 1890

erbrachten [die Ausgleichsgespräche] [...] in elf Punkten ein weitgehendes Einvernehmen. Neben kultur- und sprachpolitisch relevanten Vereinbarungen, vor allem über die Einrichtung von gesonderten Sektionen im Landeskulturrat und Landesschulrat, den Aufbau von Minderheitenschulen bei mehr als 40 andersnationalen Kindern sowie die Neugliederung der Gerichtssprengel unter nationalen Gesichtspunkten, war auch eine Änderung der Landtagsordnung durch die Trennung des Plenums in eine mit Vetorechten auszustattende deutsche und tschechische Nationalkurie in Aussicht genommen worden. ${ }^{11}$

$8 \quad$ NIEHR [Anm. 4], S. 48.

9 NIEHR [Anm. 4], S. 50.

10 Die erste Nummer erschien am 18. 8. 1857.

11 HOENSCH [Anm. 1], S. 374. 
Die Diskussionen im Landtag kosteten jedoch „wegen der jungtschechischen Opposition“12 viel Zeit, und die Ausgleichsverhandlungen wurden schließlich am 1. April 1892 „für gescheitert erklärt“. ${ }^{13}$ Wegen ihrer Brisanz waren die Wiener Punktationen in beiden untersuchten Jahrgängen (1890 und 1891) des Aussiger Anzeigers sowie der Zeitung Česká stráž allgegenwärtig und wurden in verschiedenen Rubriken erörtert und kommentiert. Obwohl sich der Diskurs zur Volkszählung offensichtlich in vieler Hinsicht auf den Diskurs zu den Wiener Punktationen bezieht, darauf reagiert und vielleicht sogar als dessen Teildiskurs bezeichnet werden könnte, beschränkt sich die vorliegende Analyse nur auf Texte, die sich explizit zur Volkszählung äußern. Diskursanalytische Untersuchungen zu den Punktationen werden Gegenstand eines selbstständigen Beitrags sein.

\section{Das Thema der Volkszählung im Aussiger Anzeiger: „Der Krieg auf dem Zählbogen"14}

Die im Zwischentitel zitierte Überschrift aus dem Aussiger Anzeiger vom 17. Januar 1891 bezieht sich auf das bewegte Geschehen rund um die Volkszählung vom 31. Dezember 1890. Sowohl die deutschsprachige als auch die tschechischsprachige Zeitung haben das Geschehen einerseits sehr intensiv reflektiert, andererseits auch vorangetrieben und eskaliert. Ins Visier wurde dabei aber nur eine einzige Spalte des Zählbogens gefasst, und zwar die „ominöse“15, „viel umstrittene[.] Spalte 11 - Umgangssprache“16. Was mit ,Umgangssprache' gemeint sei, das erklärte der Aussiger Anzeiger seinen Lesern zwei Wochen vor dem Tag der Volkszählung. In der Auffassung der Redaktion sei

[u]nter der ,Sprache im gewöhnlichen Umgange“ [...] die Sprache im Hause des Brotgebers, aber nicht die Umgangssprache am Röhrkasten oder in der Kneipe zu verstehen. [...] Es wird auch nicht gefragt, ob ob [sic!] jemand diese Sprache besser spricht als eine andere; genug, daß er sich im Hause damit verständigt. ${ }^{17}$

Diese Interpretation des Ausdrucks ,Umgangssprache“ wird allen auf die Volkszählung bezogenen Artikeln des Aussiger Anzeigers zugrunde gelegt. Andere Interpretationen werden sehr kritisch quittiert und die Redakteure des Aussiger Anzeigers plädieren sogar dafür, dass solche Interpretationen und ihnen entsprechende Handlungsweisen sanktioniert werden.

12 HOENSCH [Anm. 1], S. 374.

13 HOENSCH [Anm. 1], S. 375.

14 N. N.: Der Krieg auf dem Zählbogen. In: Aussiger Anzeiger, 17. 1. 1891.

15 N. N.: Episteln eines Volkszählungs-Kommissärs III. In: Aussiger Anzeiger, 14. 1. 1891.

16 N. N.: Episteln eines Volkszählungs-Kommissärs III. In: Aussiger Anzeiger, 14. 1. 1891. Die ,Umgangssprache' ist die im alltäglichen Umgang verwendete Sprache; das tschechische Äquivalent lautet ,obcovací řeč‘

17 N. N.: Die Volkszählung 1890. In: Aussiger Anzeiger, 17. 12. 1890. 


\subsection{Der Diskurs nach der Volkszählung: „Echte tschechische ,Patrioten' in unserem Bezirke ${ }^{\prime \prime 18}$}

Im Zusammenhang mit den Ergebnissen der Volkszählung wird im Aussiger Anzeiger das Vorgehen mehrerer Priester thematisiert, die als ihre Umgangssprache Tschechisch angegeben hatten, obwohl deren Pfarrgemeinden mehrheitlich deutschsprachig waren und die Priester daher nach der oben zitierten Auffassung des Aussiger Anzeigers als Umgangssprache Deutsch hätten angeben sollen. Beispielsweise Pater Láš, „niemand Geringerer, als der Stellvertreter unseres allverehrten und [...] derzeit wegen Erkrankung von Aussig abwesenden Herrn Dechants“, habe es angeblich „[ge]wagt[.], sich in seinem Anzeigezettel mit tschechischer Umgangssprache einzutragen“". ${ }^{19}$ Der anonyme Verfasser der Artikelserie „Episteln eines Volkszählungs-Kommissärs“ äußerte hierüber seine große Empörung und kommentierte dieses Verhalten kritisch:

$\mathrm{Da}$ dieser ,Mann des Friedens“ dies mit seiner angesehenen Stellung als Seelenhirt einer kerndeutschen Gemeinde [...] vereinbarlich gefunden hat, vermögen seine deutschen Pfarrkinder, mit welchen er doch in ihrer geliebten deutschen Muttersprache verkehrt, nicht zu fassen und schütteln unwillig die Köpfe. ${ }^{20}$

Der Verfasser wirft die rhetorische Frage auf,

[o]b wol für ein derartiges, hierstadts bereits nicht wenig Aergernis erregendes Vorgehen eines Gottesmannes die Stadtgemeinde als Kirchenpatron schweigen, und über eine derartige Provokazion unserer, den nazionalen Frieden über alles liebenden und Anders-Nazionalen gegenüber nur zusehr toleranten Bevölkerung ruhig hinwegsehen wird?21

Eine Antwort wird dem Lesepublikum zwar nicht angeboten, der Verfasser beteuert jedoch, er „wüßte wol die einzig richtige und unfehlbar wirkende Antwort, welche die Aussiger Pfarrkinder ihrem famosen Seelenhirten auf diese unerhörte Provokazion und Verletzung, ja Verhöhnung ihrer nazionalen Gefühle ertheilen sollten“. ${ }^{22}$ Die ironischen Bezeichnungen ,Mann des Friedens“ und ,famoser Seelenhirte‘ lassen auf die kritische Einstellung des Verfassers zu Pater Láš schließen, dessen Verhalten nicht nur wiederholt als ,Provokazion' bezeichnet wird (dieser an sich schon negativ wertende Ausdruck wird einmal zusätzlich noch mithilfe des Attributs ,unerhört‘ intensiviert), sondern klimatisch als eine ,Verletzung، und sogar ,Verhöhnung' der nationalen Gefühle der deutschsprachigen Bevölkerung Aussigs. Der Verfasser versteht also das Ausfüllen des Zählbogens -

18 N. N.: Echte tschechische „Patrioten“ in unserem Bezirke. In: Aussiger Anzeiger, 14. 1. 1891.

19 N. N.: Episteln eines Volkszählungs-Kommissärs II. In: Aussiger Anzeiger, 10. 1. 1891. Hervorhebung im Original gesperrt.

20 N. N.: Episteln eines Volkszählungs-Kommissärs II. In: Aussiger Anzeiger, 10. 1. 1891.

21 N. N.: Episteln eines Volkszählungs-Kommissärs II. In: Aussiger Anzeiger, 10. 1. 1891.

22 N. N.: Episteln eines Volkszählungs-Kommissärs II. In: Aussiger Anzeiger, 10. 1. 1891. 
bzw. zumindest der Spalte 11 (Umgangssprache) - nicht als eine private Angelegenheit, sondern als eine öffentliche Tat, die Pater Láš als Pfarrverweser und somit Person des öffentlichen Lebens nicht nach seinem eigenen Willen und Gewissen ausführen darf.

Demselben Argumentationsmuster begegnen wir im Zusammenhang mit einer anderen Person des öffentlichen Lebens, dem in Steben [Stebno] bei Aussig tätigen Pfarrer Adalbert Schneider. Unter dem Titel „Echte tschechische ,Patrioten“ in unserem Bezirke“ berichtete der Aussiger Anzeiger, dass „das leuchtende Beispiel unseres Pfarradministrators [...] Nachahmung gefunden [hat].“ Pater Schneider habe - „als Seelsorger einer reindeutschen Gemeinde“ - in dem Zählbogen ebenfalls „böhmisch“ als seine Umgangssprache angegeben. Dies wird als Äußerung einer „stramm tschechischnazionalen Gesinnung“ interpretiert. „Doch nicht genug daran; er [P. Schneider] gieng in der Bethätigung seiner stramm tschechischnazionalen Gesinnung noch weiter, indem er überdies auch sein Gesinde als ein mit tschechischer Umgangssprache verkehrendes eintrug. “23

Der Stebener Pfarrer reagierte vermutlich in einem Schreiben auf diesen Artikel, seine Reaktion wurde in der Zeitung jedoch nicht abgedruckt. Stattdessen erschien am 21. Januar in der Rubrik „Briefkasten der Schriftleitung“ eine sehr despektierliche Antwort der Redaktion auf das Schreiben Pater Schneiders. Dass der Pfarrer wohl die Veröffentlichung seines Briefes in der Zeitung erwartete, sei - so hieß es in der Antwort der Redaktion - eine „geradezu lächerliche Zumutung“ gewesen. Die Redaktion nannte den Brief des Pfarrers „sinnloses Geschreibsel“ sowie „uns [der Redaktion] völlig unverständliches Gesalbader" und begründete, warum sie den Brief überhaupt einer Antwort wert erachtete:

Wenn wir von Ihrem sinnlosen Geschreibsel - in welchem Sie, die von uns gebrachte Thatsache, daß Sie es für gut befunden haben, sich als Seelsorger einer reindeutschen Gemeinde mit tschechischer Umgangssprache einzutragen, gleichwol zugeben, uns aber die geradezu lächerliche Zumutung stellen, das uns völlig unverständliche Gesalbader in unserem Blatte zu veröffentlichen - überhaupt Notiz nehmen, so geschieht dies nur, um Sie darüber aufzuklären, daß Sie hinsichtlich der Urheberschaft der diesbezüglichen Notiz in einen argen Irrtum befangen sind [...]. Zu Ihrer Beruhigung diene Ihnen somit, daß unsere Notiz lediglich auf Grund einer uns diesbezüglich von Steben zukommenen einfachen Mittheilung der beregten Thatsache des Verhaltens eines Priesters auf den Höhen unseres kerndeutschen Mittelgebirges, in unserer Redakzion verfaßt wurde [...]. ${ }^{24}$

Dieser emotiven Reaktion des Aussiger Anzeigers ist zum einen zu entnehmen, dass die Kritik in dem Artikel „Echte tschechische ,Patrioten“ in unserem Bezirke“ lediglich auf einer der Redaktion „von Steben zukommenen einfachen Mittheilung“ basiert und durch keine weiteren Beweise gestützt ist. Es ist übrigens der einzige Fall, in welchem das Lesepublikum wenigstens vage Hinweise über den Ursprung der angeblichen Informationen aus den Zählbögen der im Blatt kritisierten Personen erhielt. In den anderen

23 N. N.: Echte tschechische „Patrioten“ in unserem Bezirke. In: Aussiger Anzeiger, 14. 1. 1891.

24 N. N.: Herrn P. Adalbert Schneider in Steben! In: Aussiger Anzeiger, 21. 1. 1891. 
bereits oder im Folgenden zitierten Fällen finden sich keinerlei Aufschlüsse über die Quelle der präsentierten Informationen. Ob sie etwa von Vertrauensmännern verraten wurden, deren Mitwirkung an der Volkszählung zwei Wochen vor dem Stichtag der Volkszählung im Aussiger Anzeiger empfohlen wurde ${ }^{25}$, oder ob sie einer anderen Quelle entstammten, kann heute kaum geklärt werden.

Zum anderen geht aus dem Text hervor, dass die Redaktion im Falle des Stebener Pfarrers dasselbe Argument anbrachte, das sie im Falle des Stadtpfarrers P. Láš äußerte und das sich auf der eingangs zitierten Auffassung gründet, die Umgangssprache sei diejenige Sprache, die eine Person im alltäglichen Umgang benutzt. Da P. Schneider angeblich „Seelsorger einer reindeutschen Gemeinde“ war, hätte er demnach als Umgangssprache Deutsch eintragen müssen. Problematisch bleibt jedoch das Attribut ,reindeutsch“, das in dem Text durch keine Angaben gestützt wird, ebensowenig wie etwa das Attribut ,kerndeutsch“ in den Verbindungen „auf den Höhen unseres kerndeutschen Mittelgebirges“26, „Seelenhirt einer kerndeutschen Gemeinde“27 oder z. B. „unsere kerndeutsche Vaterstadt" ${ }^{\text {28 }}$.

Das im Aussiger Anzeiger harsch kritisierte Verhalten des Aussiger und des Stebener Pfarrers scheint ein Impuls gewesen zu sein, warum bereits in der nächsten Nummer nach der Veröffentlichung der Reaktion auf den Brief Pater Schneiders ein Artikel mit dem Titel „Die Geistlichkeit und die Volkszählung“ erschien, sogar auf der Titelseite. „Fast aus allen deutschen Gegenden“ werde berichtet, „daß es gerade die tschechischen Priester, hauptsächlich die jüngeren Geistlichen sind, welche in geradezu herausfordernder Weise sich zur tschechischen Umgangssprache bekannten. "29 Die Bedeutung der bereits zitierten Ausdrücke ,reindeutsch“ in Bezug auf ,Gemeinde“ oder ,kerndeutsch“ in Bezug auf ,Gemeinde“, ,Vaterstadt' und ,Mittelgebirge“ kommt hier in Pauschalformulierungen zum Vorschein wie z. B. „aus allen deutschen Gegenden“ oder „[i]n Orten, wo nur deutsch gesprochen wird, wo überhaupt keine Tschechen wohnen“":30

In Orten, wo nur deutsch gesprochen wird, wo überhaupt keine Tschechen wohnen, hatten tschechische Priester die Unverfrorenheit, „tschechisch“ als ihre Umgangssprache anzugeben. Die frommen Herren haben damit eine offenkundige Unwahrheit gesagt, doch das kümmert sie wenig; zur größeren Ehre der tschechischen Nazion kommt es ihnen auf eine Unwahrheit mehr oder weniger nicht an. ${ }^{31}$

Solche Pauschalurteile werden in der Zeitung durch keine Beweise gestützt. Außerdem kann es triftige Beweise wohl kaum gegeben haben, da der einzige schriftliche Beweis,

25 Vgl. Kapitel 3.3.

26 N. N.: Herrn P. Adalbert Schneider in Steben! In: Aussiger Anzeiger, 21. 1. 1891.

27 N. N.: Episteln eines Volkszählungs-Kommissärs II. In: Aussiger Anzeiger, 10. 1. 1891.

28 N. N.: Episteln eines Volkszählungs-Kommissärs III. In: Aussiger Anzeiger, 14. 1. 1891.

29 N. N.: Die Geistlichkeit und die Volkszählung. In: Aussiger Anzeiger, 24. 1. 1891.

30 N. N.: Die Geistlichkeit und die Volkszählung. In: Aussiger Anzeiger, 24. 1. 1891.

31 N. N.: Die Geistlichkeit und die Volkszählung. In: Aussiger Anzeiger, 24. 1. 1891. Hervorhebung im Original gesperrt. 
auf dessen Grundlage die Zuordnung einer Person zu einer nationalen Gruppe erfolgen könnte (,Deutsche“/,deutsch' oder ,Tschechen'/,tschechisch'), gerade der Zählbogen mit der Spalte zur Umgangssprache gewesen sein dürfte. Dies ist jedoch gleich aus mehreren Gründen problematisch: Zum einen wurde die Umgangssprache erst ab 1880 erfasst, ${ }^{32}$ zum anderen ist es fraglich, ob allein die Umgangssprache einer Person als Kriterium für die Zuordnung dieser Person zu einer nationalen Gruppe herhalten kann. Schließlich ist zu beachten, dass es objektive Kriterien zu einer richtigen bzw. unanfechtbaren Bestimmung der Umgangssprache kaum gibt, wie nicht zuletzt aus dem Diskurs in der Presse hervorgeht.

Dass - wie vom Aussiger Anzeiger behauptet wird - viele Priester als ihre Umgangssprache Tschechisch angaben, wird in dem Artikel als eine „Unverfrorenheit“"33 bezeichnet und somit allein schon durch diesen Ausdruck dezidiert negativ interpretiert. AuBerdem ist der Verfasser überzeugt, dass die Volkszählung „wieder einmal den Beweis erbracht [hat], daß die tschechische Geistlichkeit ultra-nazional gesinnt ist, daß sie bei jeder Gelegenheit für das Tschechenthum eintritt.“ Obwohl das Adjektiv ,national“ in vielen Kontexten positiv konnotiert ist, erhält es in diesem Kontext eine pejorative Konnotation, zum einen durch das Konfix ,ultra-', welches ein Übermaß signalisiert, zum anderen dadurch, dass es hier zur Kennzeichnung derjenigen nationalen Gruppe dient, von der sich die Redaktion des Aussiger Anzeigers konsequent abzugrenzen versucht. Die Stadt Aussig gehört zur Leitmeritzer Diözese [Litoměřice], welche in dem Artikel als eine Diözese „mit überwiegend deutscher Bevölkerung“ bezeichnet wird. Auch in solchen Diözesen werde jedoch „das Tschechenthum im Klerus bevorzugt. Wir sehen tschechische Heißsporne an rein deutschen Pfarrgemeinden, und alle Beschwerden der Pfarrkinder haben es nicht vermocht, eine Besserung herbeizuführen." ${ }^{4}$ Als Lösung dieser unerwünschten Lage wird Folgendes vorgeschlagen:

Aufgabe des deutschen Volkes wäre es, sich die tschechischen Priester nicht länger mehr aufdrängen zu lassen. Das Verhalten des tschechischen Klerus bei der Volkszählung sollte von den deutschen Diözesanen nicht ruhig hingenommen werden. In einer Massenkundgebung sollte dem Leitmeritzer Dom gesagt werden, daß die deutschen Katholiken auch deutsche Seelsorger wünschen [...] Es muß überhaupt gefordert werden, daß die Leitung einer vorwiegend deutschen Diözese eine deutsche sei, daß somit auch im Priesterseminar ein deutscher, auf keinen Fall aber ein tschechischer Geist vorherrsche. [...] «35

Trotz der Verachtung, mit welcher das Vorgehen des Aussiger und des Stebener Pfarrers in der Zeitung präsentiert wurde, wurde von der Redaktion keine konkrete Sanktionierung vorgeschlagen. Dasselbe gilt auch für „einige hiesige k. k. Beamten“, die es angeblich „als ihre ,patriotische Pflicht“ betrachtet [hatten], sich in der Rubrik 11 gleichfalls

32 Vgl. TEIBENBACHER [Anm.2].

33 N. N.: Die Geistlichkeit und die Volkszählung. In: Aussiger Anzeiger, 24. 1. 1891.

34 N. N.: Die Geistlichkeit und die Volkszählung. In: Aussiger Anzeiger, 24. 1. 1891.

35 N. N.: Die Geistlichkeit und die Volkszählung. In: Aussiger Anzeiger, 24. 1. 1891. Alle Hervorhebungen im Original gesperrt. 
mit ,böhmisch“ resp. tschechisch einzutragen“. Später sollen diese „famosen ,Vlastenci““ (Patrioten) die Angabe korrigiert haben, und zwar so, dass sie das Wort „deutsch“ eintrugen, mit der Ergänzung ,jedoch unter Wahrung der tschechischen Nazionalität.“ Dazu bemerkt der Aussiger Anzeiger ironisch: „[...] als ob sie [...] darum befragt worden wären. - Doch gönnen wir ihnen das Vergnügen. “36 Die angeblichen Einträge der nicht weiter spezifizierten „hiesigen k. k. Beamten“ in den Zählbögen scheinen zu implizieren, dass diese Beamten ,Umgangssprache“ als ,die Sprache im gewöhnlichen Umgange ${ }^{\text {‘37 }}$ verstanden, sie dabei vielleicht nicht notwendigerweise mit ,Muttersprache“ gleichsetzten (wobei aber ihre Umgangssprache auch identisch mit ihrer Muttersprache gewesen sein könnte) und dass sie außerdem ihre nationale Selbstidentifikation nicht an die Umgangssprache gebunden sahen.

Während also das Vorgehen sowohl der beiden Pfarrer als auch der k. k. Beamten kritisiert wird, ohne dass aber Sanktionen gefordert würden, so wird etwa im Falle des Bräuhauspächters von Schöbritz [Všebořice, heute ein Stadtteil von Aussig] eine konkrete Sanktionierung befürwortet, und als Vorbild wird dem Lesepublikum das Verhalten „eine[r] nicht unerhebliche[n] Anzahl von Abnehmern des Schöbritzer Bieres“ vor Augen geführt:

\begin{abstract}
Nun, eine nicht unerhebliche Anzahl von Abnehmern des Schöbritzer Bieres haben daraufhin es nicht unterlassen, auch ihren Standpunkt in unzweideutiger Weise zu markieren und heraus die Konsequenzen durch Lösung ihres bisherigen Verhältnisses zum Schöbritzer Bräuhause zu ziehen, indem sie sich die Frage vorlegten, in welcher anderen Sprache als der deutschen sie nun mit ihrem bisherigen Bierlieferanten Umgang und Verkehr pflegen könnten, da sie der „seinigen“ bisher noch keineswegs mächtig sind. ${ }^{38}$
\end{abstract}

Ebenfalls das Verhalten des Trebnitzer [Třebenice] Tierarztes Franz Steckl, der bei der Volkszählung angeblich Tschechisch als Umgangssprache angegeben hatte, soll nach Meinung der Redaktion nicht ohne Sanktionen bleiben. Es wurden bereits „[v]on deutscher Seite [...] in Folge dessen Anstalten getroffen, einen deutschen Thierarzt zu bewegen, sich in Trebnitz niederzulassen“. Dies befürwortet die Redaktion des Aussiger Anzeigers, nennt ein solches Vorgehen „vollkommen gerechtfertigt“ und betont, dass es „nur zu wünschen [ist], daß das Bestreben ,Kauft nur bei Deutschen, unterstützt nur Deutsche“ ein allgemeines werde, damit die Tschechen sehen, wer in unserer Stadt ,maßgebend" und wer ,abhängig' ist." Neben dem Tierarzt Franz Steckl sollte auch dessen Bruder als „Pächter des einzigen für Bälle, Konzerte und sonstig größeren Unterhaltungen passenden Lokales“ mit Strafmaßnahmen rechnen: Dem Aussiger Anzeiger zufolge hätten „alle deutschen Vereine beschlossen, für heuer von allen Bällen abzusehen und sich nur mit kleineren Unterhaltungen zu begnügen“. 39

36 N. N.: Episteln eines Volkszählungskommissärs III. In: Aussiger Anzeiger, 14. 1. 1891.

37 Vgl. Kap. 3.

38 N. N.: Echte tschechische „Patrioten“ in unserem Bezirke. In: Aussiger Anzeiger, 14. 1. 1891.

39 N. N.: Von der Trebnitzer Sprachgrenze. In: Aussiger Anzeiger, 17. 1. 1891. 
Die Frage, welche Umgangssprache eine Person bei der Volkszählung im Anzeigezettel angegeben hatte, taucht auch in solchen Artikeln auf, in deren Vordergrund ein anderes Thema als die Volkszählung steht. So berichtet ein Artikel mit dem Titel „Versetzung“ über einen „Beamte[n] der A. T. E. Herr[n] Resač, der durch mehrere Jahre auch in Aussig als Verkehrsbeamte [sic!] stazioniert war und sonach unseren Lesern bestens bekannt ist". Herr Resač

wurde im Sommer v. J. bekanntlich zum Stazionschef in Komotau [Chomutov] befördert. Nun geht uns die Mittheilung zu, daß Herr Resač plötzlich von Komotau nach Bilin [Bílina] versetzt wurde. Bemerkt sei, daß der Herr Stazionschef Resač bei der letzten Volkszählung sich mit tschechischer Umgangssprache in den Anzeigezettel eingezeichnet hat, während er vor 10 Jahren sich mit deutscher Umgangssprache bekannte. ${ }^{40}$

Unklar bleibt, ob der Verfasser mit dem letzten Satz implizieren wollte, dass die plötzliche Versetzung mit dem Eintrag der Umgangssprache einen Zusammenhang gehabt haben könnte, oder ob die Information zur Umgangssprache nur als eine zusätzliche Angabe intendiert war, die keinen Bezug zu der Versetzung hatte.

\subsection{Die Ergebnisse der Volkszählung}

In Anbetracht der konsequenten Hervorhebung der deutschen Umgangssprache im Aussiger Anzeiger, die damit begründet wird, dass die Stadt Aussig „kerndeutsch“ sei, ${ }^{41}$ genauso wie die Ortschaften des Böhmischen Mittelgebirges, ${ }^{42}$ und der Umgang daher in keiner anderen Sprache als der deutschen gepflegt werden könne, verwundert es nicht wenig, dass in mehreren Artikeln mit dem Titel „Volkszählungs-Ergebnisse“ von ,Umgangssprache“ oder ,Nationalität' keine Rede ist. Es werden in diesen Artikeln lediglich Gemeinden aufgelistet mit der Angabe der Einwohnerzahl von 1880 und von 1890. Diese Übersicht wird nur mit einem schlichten Satz eingeleitet und nicht weiter kommentiert: „Von den vorläufigen Ergebnissen der Volkszählung am 31. Dezember theilen wir weiter nachstehende mit: [...]“.43 Identisch eingeleitet ist auch etwa die Übersicht vom 21. Januar, die aber Angaben aus anderen Ortschaften enthält.

Bereits am 14. Januar erschien ein Artikel, der sich zu den Ergebnissen der Volkszählung in der Stadt Aussig selbst äußert und die Umgangssprache sowie Nationalität ebenfalls völlig außer Acht lässt:

[...] Die Einwohnerzahl ist seit dem Jahre 1880, in dem man 16524 Personen zählte, um 7188

Seelen gestiegen [...] Das Resultat der Volkszählung ist sicherlich ein ungemein erfreuliches,

40 N. N.: Versetzung. In: Aussiger Anzeiger, 24. 1. 1891.

41 Vgl. z. B. N. N.: Episteln eines Volkszählungs-Kommissärs III. In: Aussiger Anzeiger, 14. 1. 1891.

42 Vgl. z. B. N. N.: Herrn P. Adalbert Schneider in Steben! In: Aussiger Anzeiger, 21. 1. 1891.

43 N. N.: Volkszählungs-Ergebnisse. In: Aussiger Anzeiger, 17. 1. 1891. 
und wenn auch jene Ziffer, die man allgemein erwartet hatte, nämlich 25000 nicht erreicht wurde, so hat doch das Ergebnis der Zählung den immensen Aufschwung unserer Vaterstadt in ganz unzweideutiger Weise dargethan. [...] und weiters darf nicht übersehen werden, daß eine sehr große Zahl der in Aussig in Arbeit stehenden Personen in den mit unserer Vaterstadt gleichsam zusammenhängenden Orten Kleischa [Klíše], Pockau [Bukov], Schönpriesen [Krásné Březno], etc. wohnen und weisen diese Orte, eine ganz unglaubliche Bevölkerungszunahme auf. [...] Wir können immerhin mit dem Resultate zufrieden sein und möchten nur wünschen, daß unsere Vaterstadt auch noch fernerhin wachse, blühe und gedeihe. ${ }^{44}$

Dieser Darstellung ist zu entnehmen, dass der Verfasser den Anstieg der Bevölkerung allgemein als erstrebenswert betrachtete und die Umgangssprache oder Nationalität der Bewohner nicht für ausschlaggebend hielt. Die Wortwahl des Verfassers fällt wiederholt zugunsten des emotional positiv besetzten Ausdrucks ,Vaterstadt', nicht des neutralen Konkurrenzausdrucks ,Stadt‘. Die Verbindung, immenser Aufschwung ist ebenfalls ausgeprägt positiv konnotiert und die völlige Ausklammerung der Kategorie ,Umgangssprache zeugt davon, dass der Verfasser lediglich allgemein das Wachstum und die Blüte Aussigs für wesentlich erachtete, unabhängig davon, ob der Zuwachs primär Menschen mit deutscher Umgangssprache oder solchen mit tschechischer Umgangssprache zu verdanken war.

Ergebnisse der Volkszählung hinsichtlich Umgangssprache bzw. Nationalität wurden aber nach wie vor in anderen Kontexten thematisiert. So weiß ein kurzer Artikel vom 17. Januar 1891 Folgendes zu berichten:

Bei der Volkszählung in Kolloletsch [Kololeč] (Bez. Lobositz [Lovosice]) wurden die deutschen Zählungskommissär thätlich angegriffen und mußten sich flüchten, bis Deutsche zu Hilfe kamen. Trotz des tschechischen Terrorismus wird das Zählungsergebnis für die Deutschen günstiger als vor zehn Jahren ausfallen. Gegen die Thäter wurde die Strafanzeige erstattet. ${ }^{45}$

Details zu dem Geschehen in Kolloletsch und den genauen Umständen des Vorfalls bringt die Zeitung allerdings nicht.

\subsection{Der Diskurs vor der Volkszählung: „überall und überall nur Tschechen“46}

Im Hinblick auf die große Bedeutung, die den Ergebnissen der Volkszählung von der Redaktion des Aussiger Anzeigers besonders in den ersten Wochen nach dem Stichtag der Volkszählung offensichtlich beigemessen wurde, bietet sich die Frage, ob bzw. inwiefern die Redaktion auch bereits vor der Volkszählung versuchte, auf die Meinungen, Einstellungen und somit auf das Verhalten der Leser bei dem Zensus Einfluss zu nehmen. Anders als in der Zeitung Česká stráž erscheint im Aussiger Anzeiger der erste, explizit auf

44 N. N.: Das Ergebnis der Volkszählung in Aussig. In: Aussiger Anzeiger, 14. 1. 1891.

45 N. N.: Bei der Volkszählung in Kolloletsch. In: Aussiger Anzeiger, 17. 1. 1891.

46 N. N.: Die Volkszählung 1890. In: Aussiger Anzeiger, 17. 12. 1890. 
die Volkszählung bezogene Artikel erst zwei Wochen vor dem Stichtag, nämlich am 17. Dezember 1890. Der umfangreiche Artikel „Die Volkszählung 1890“ ist in 14 Abschnitte geteilt und mit Zwischentiteln versehen. Der einleitende Absatz steht ohne Zwischentitel und die weiteren Abschnitte, die zumeist aus mehreren Absätzen bestehen, tragen folgende Zwischentitel: „Die Art der Zählung“, „Vertrauensmänner“, „Der Zählungskommissär“, „Das Ausfüllen der Anzeigezettel“, „Gemeindeübersicht und Zählungsbuch“, „Gebraucht Euere Rechte!“, „Tschechische Absichten“, „Drohende Gefahren“, „Die beste Abwehr“, „Was ist ,Umgangssprache?‘“, „Weitere Versichtsmaßregeln“, „Was also alles zu beachten ist" und „Schlußwort“.

In der Einleitung und im Abschnitt „Die Art der Zählung“ wird der Verlauf des Zensus dargelegt. Entweder erhält ,jeder Familienvorstand einen Anzeigezettel mit eingedruckten Fragen zu eigener Ausfüllung“ oder es kommt „ein von der Gemeinde ernannter Zählungskommissär“, welcher „den Parteien die Angaben mündlich abfordert und selbst in einen sogenannten Aufnahmsbogen einträgt." ${ }^{47}$ Die erste Variante betraf diejenigen Gemeinden, die „das Zählungsgeschäft zur Gänze selbst besorgen“ sowie einen Teil derjenigen, „welche die k. k. Bezirkshauptmannschaft dazu bestimmt“. ${ }^{48}$ In allen anderen Gemeinden waren Zählungskommissäre tätig. Neben den von amtlicher Seite ernannten Personen wird die Bestimmung von „Vertrauensmännern“ dringend empfohlen. Dies sollen Freiwillige oder „von der Vertretung damit [B]etraut[e]“ sein, welche

das Gesetz und diese Belehrungen in vorhinein [...] studiren und alles dasjenige in Vorschlag [...] bringen, was die örtlichen Verhältnisse zum Vortheile der Gemeinde und der deutschen Sache erfordern. Es wäre gefehlt, wenn die deutschen Gemeinden sich in jedem Falle nur auf die angestellten Organe verlassen wollten. ${ }^{49}$

Diese Maßnahmen seien deshalb unerlässlich, weil die Volkszählung „eine[.] so unendlich wichtige[.] Sache“50 sei. In den Abschnitten „Das Ausfüllen der Anzeigezettel“ und „Gemeindeübersicht und Zählungsbuch“ werden die Leser ausdrücklich auf ihr Recht aufmerksam gemacht, die Angaben in den Zählungsbüchern einzusehen und eventuell zu korrigieren. Überdies habe der Gemeindevorsteher die Pflicht, die Richtigkeit der Anzeigezettel zu überprüfen: „Das Gesetz wünscht sogar, daß er eine Ueberprüfung von Haus zu Haus oder wenigstens in einzelnen ausgewählten Fällen vornehme. “51 In dem Abschnitt „Gebraucht Euere Rechte!“ werden die Auswirkungen der Volkszählung dargelegt und mit einem Appell an das Lesepublikum verbunden:

Es ist äußerst notwendig, gerade bei der bevorstehenden Zählung, daß die deutsche Bevölkerung von allen diesen Rechten Gebrauch mache. Denn durch eine den Thatsachen entspre- 
chende Abgrenzung des deutschen und tschechischen Gebietes, sowie durch entsprechende Einrichtung der Verwaltungsanstalten wollen wir Deutschen ja den Frieden zwischen beiden Stämmen des Landes auf einer dauerhaften Grundlage herstellen. Jeder Theil soll in seinem Gebiete ungestört leben dürfen, keiner aber den anderen beherrschen. Während wir Deutschen für dieses Streben nun alles einsetzen und zu jedem Opfer bereit sind, wollen aber die Jungtschechen, welche gegenwärtig über die Mehrheit der tschechischen Volksvertreter im Landtage verfügen, von Freiheit und Frieden nichts wissen. Sie verlangen auf Grund eines „böhmischen Staatsrechtes“, das wir als österreichische Staatsbürger nicht kennen, die Herrschaft über ganz Böhmen, sie wollen uns überall Beamte und Richter ihrer Sprache und für jene ihrer Arbeitskräfte, die bei deutschen Unternehmungen ihr Brot finden, Schulen ihrer (d. h. der tschechischen) Sprache aufzwingen. ${ }^{52}$

Diese Befürchtungen deutscherseits scheinen im öffentlichen Diskurs seit Ende der sechziger Jahre des 19. Jahrhunderts präsent gewesen zu sein. Etwa in der südmährischen Presse traten sie beispielsweise in der Auseinandersetzung um die Tilgung des Titels ,königlich“ aus der offiziellen Bezeichnung der Stadt Znaim [Znojmo] zutage, welche eigentlich ein Streit darüber war, „ob sich die Znaimer Bevölkerung eine staatsrechtliche Organisation wünscht, die die ,alten historischen Rechte ${ }^{63}$ der Länder der böhmischen Krone berücksichtigt und sich somit den Forderungen eines Großteils tschechischer Politiker nach mehr Autonomie für Böhmen, Mähren und Schlesien anschließt“. ${ }^{4}$ Der Artikel „Die Volkszählung 1890“ im Aussiger Anzeiger scheint ähnliche staatsrechtliche Befürchtungen auszudrücken.

Das Unbehagen wird weiter im Abschnitt „Tschechische Absichten“ spezifiziert. Die tschechischen Zeitungen hätten früher tschechische Handwerker und Tagelöhner für „böhmische[.] Unternehmen in deutscher Gegend“55 gelockt:

[...] jetzt soll die Volkszählung ihre Anwesenheit selbst beurkunden, und durch die Dienstboten, Taglöhner und Lehrlinge, die oft mit aufgehobenen Händen beim deutschen Arbeitgeber Dienst und Brot gesucht, soll jetzt der Beweis geliefert werden, daß es einen deutschen Volksstamm in Böhmen gar nicht gebe, sondern überall und überall nur Tschechen und mitten drin ein paar Deutsche. ${ }^{56}$

Dem Aussiger Anzeiger zufolge beabsichtigen die Tschechen, den Beweis zu liefern, „daß wir in Böhmen nur ein tschechisches und ein gemischtes Gebiet haben“ und zu diesem

52 N. N.: Die Volkszählung 1890. In: Aussiger Anzeiger, 17. 12. 1890.

53 KANN, Robert A.: Das Nationalitätenproblem der Habsburgermonarchie. Geschichte und Ideengehalt der nationalen Bestrebungen vom Vormärz bis zur Auflösung des Reiches im Jahre 1918. 1. Bd. Graz, Köln 1964, S. 149.

54 PAVLÍČKOVÁ, Tereza: Die Entwicklung des Nationalitätenkonflikts in der Znaimer deutschen Presse 1850-1938. Olomouc 2013, S. 84.

55 N. N.: Die Volkszählung 1890. In: Aussiger Anzeiger, 17. 12. 1890.

56 N. N.: Die Volkszählung 1890. In: Aussiger Anzeiger, 17. 12. 1890. 
Zweck „rüsten [sie] sich wie zu einem Feldzuge zu dem sonst in aller Welt so friedlichen Werke einer Volkszählung“. ${ }^{57}$

\section{Das Thema der Volkszählung in der Zeitung Česká stráž: "die Verteidigung des uralten tschechischen nationalen Besitzstandes"}

Während der Aussiger Anzeiger Sanktionen für den Trebnitzer Tierarzt Franz Steckl forderte, weil er sich bei der Volkszählung mit tschechischer Umgangssprache gemeldet hatte (es wurden „Anstalten getroffen, einen deutschen Thierarzt zu bewegen, sich in Trebnitz niederzulassen" ${ }^{59}$ und Sanktionen wirtschaftlicher Art wurden sogar in Bezug auf Steckls Bruder in Gang gesetzt), schreibt die Zeitung Česká stráž in dem im Zwischentitel zitierten Artikel, dass „gegen die deutsche Angriffs- und Kampflust das beste Mittel die feste, unverwüstliche Zähigkeit der slawischen und tschechischen Natur"60 sei. Diese Eigenschaften seien bei den Trebnitzern in Fülle zu finden, insbesondere in der Persönlichkeit des Trebnitzer Bürgermeisters MUDr. Pařík. Im Hinblick auf die Aktivitäten des Trebnitzer Schutzvereines Germania beruft sich die Zeitung Česká stráž auf eine angeblich kritische, jedoch nicht näher spezifizierte Äußerung des Grafen Ledebur im Landtag und konstatiert, dass dank dieser Kritik Ledeburs an dem Verein Germania die Trebnitzer Tschechen wissen würden, mit wem sie es zu tun hätten, und „ihre Energie für die Verteidigung des uralten tschechischen nationalen Besitzstandes [...] wird sicherlich ihre Pflicht tun."61

Trebnitz wird in der Zeitung Česká stráž als ein „tapferes tschechisches Bollwerk“ bezeichnet, in welchem „einer der denkbar schwersten nationalen Kämpfe“62 geführt werde. Zur Unterstützung dieser These werden Zahlen angeführt, die jedoch durch keine Beweise gestützt sind: In Trebnitz würden 2000 Tschechen und 500 Deutsche leben. ${ }^{63}$ Diejenigen Zahlen der Trebnitzer Bevölkerung, die sich angeblich aus einer Probezählung im Zusammenhang mit der Volkszählung ergaben und im Aussiger Anzei-

57 N. N.: Die Volkszählung 1890. In: Aussiger Anzeiger, 17. 12. 1890.

58 N. N.: „Síla německého ducha." In: Česká stráž, 21. 6. 1890: „uhájení prastarého národního majetku českého“. Hervorhebung im Original gesperrt. Alle Zitate aus der tschechischen Zeitung werden in der von mir vorgenommenen deutschen Übersetzung angeführt; das tschechische Original findet sich in der Fußnote.

59 N. N.: Von der Trebnitzer Sprachgrenze. In: Aussiger Anzeiger, 17. 1. 1891.

60 N. N.: „Síla německého ducha.“ In: Česká stráž, 21. 6. 1890: „Proti útočnosti a rvavosti německé nejlepším prostředkem jest tuhá nezmarná houževnatost slovanské a české povahy [...]“.

61 N. N.: „Síla německého ducha.“ In: Česká stráž, 21. 6. 1890: „Když tedy o „Germanii‘ a jejích cílech z úst takových padl soud tak zdrcující, pak nebudou Čechové třebeničtí dlouho v rozpacích, koho mají před sebou a jejich energie k uhájení prastarého národniho majetku českého [...] dojista svou povinnost vykoná“.

62 N. N.: „Síla německého ducha.“ In: Česká stráž, 21. 6. 1890: „Na statečné české baště Třebenicích vede se jeden z nejtě̌̌ších zápasů národních, jaký si vůbec lze mysliti.“ Hervorhebung im Original gesperrt.

63 Vgl. N. N.: „Síla německého ducha.“ In: Česká stráž, 21. 6. 1890: „Po jejich chuti [po chuti ,milých našich súsedů Deutschböhmů] by ovšem bylo, aby 2000 Čechů třebenických podrobilo se 500 Němců a aby tito mohli pak říci, že Třebenice je město ryze německého rázu." 
ger angeführt werden, unterscheiden sich von den Angaben der tschechischen Zeitung diametral: „Zur Freude aller Gutgesinnten ergab eine Probezählung ca. 600 Deutsche, gegenüber 800 Tschechen, während die Volkszählung vor 10 Jahren 300 Deutsche und 1100 Tschechen auswies. “64 Dies sei den „vielen Bemühungen des hiesigen Schutzvereines Germania“ zu verdanken, der somit die Bestrebungen der „tschechischen Machthaber“ vereitelte, welche angeblich „gelegentlich der Volkszählung allen ihren Terrorismus aufgeboten [haben], um die deutsche Partei in verschwindender Minderheit erscheinen zu lassen." ${ }^{65}$ Die vom Aussiger Anzeiger angegebenen Zahlen ergeben außerdem die Summe 1400 (sowohl für das Jahr 1890 als auch für 1880), während die Gesamtbevölkerung der Gemeinde Trebnitz laut Česká stráż 2500 beträgt, also um 1100 Personen mehr im Vergleich zu der Angabe des Aussiger Anzeigers.

Die Persönlichkeit des Aussiger Pfarrverwesers P. Láš, dessen Vorgehen bei der Volkszählung im Aussiger Anzeiger großen Unwillen erregt hatte und in der feuilletonistischen Artikelserie „Episteln eines Volkszählungs-Kommissärs“ thematisiert wurde, fand ihren Weg auf die Seiten der Zeitung Česká stráž bereits im Juni 1890, also ein halbes Jahr vor dem Stichtag der Volkszählung, auf der sich dieser Priester angeblich zur tschechischen Umgangssprache bekannte, obwohl er - aus der Sicht des Aussiger Anzeigers - vorwiegend mit der deutschsprachigen Bevölkerung Umgang pflegte und daher als Umgangssprache „Deutsch“ hätte angeben sollen. Unter Berufung auf die Rumburger Zeitung schreibt die Zeitung Česká stráż am 21. Juni 1890, dass der „Hetzkaplan“ - so angeblich der Ausdruck der Rumburger Zeitung - nach Hodkowitz [Hodkovice] versetzt werden soll, da er sich in Aussig zur tschechischen Umgangssprache bekannte und somit eine Provokation begangen habe. Unklar bleibt dabei, bei welcher Gelegenheit sich Pater Láš zur tschechischen Umgangssprache bekannte, wenn als Stichtag der Volkszählung erst der 31. Dezember 1890 galt, und warum der Aussiger Anzeiger erst im Januar 1891 diesen Fall zur Sprache brachte. Česká stráž nennt das Vorgehen Pater Lášs „tapfer“ und stellt fest, dass Hodkowitz wohl ein vorwiegend tschechischer Ort sei, dass aber trotzdem „Feuer auf dem deutschböhmischen Dache"66 brenne.

Da sich dem Aussiger Anzeiger zufolge sogar mehrere Priester bei der Volkszählung ähnlich wie Pater Láš verhielten, forderte das Blatt, dass die deutschen Gläubigen von der Leitung der Leitmeritzer Diözese deutsche Seelsorger verlangen. ${ }^{67}$ Der Zeitung Česká stráž zufolge gebe es jedoch „nicht so viele Priester deutscher Nationalität in Böhmen, als dass man diesen deutschen Gelüsten entgegenkommen könnte, selbst wenn man möchte." ${ }^{68}$ Die tschechische Zeitung äußert ihren Unwillen darüber, dass von den tschechischen Priestern ein Opfer verlangt werde, welches in rein tschechischen Gebieten nicht einmal von „dem untertänigsten Diener deutscher Sprache“ verlangt werde: ,[E]r

64 N. N.: Von der Trebnitzer Sprachgrenze. In: Aussiger Anzeiger, 17. 1. 1891.

65 N. N.: Von der Trebnitzer Sprachgrenze. In: Aussiger Anzeiger, 17. 1. 1891.

66 N. N.: Štvanice na českého kněze. In: Česká stráž, 21. 6. 1890: „Nyní, kdy se má dostati do místa dle všeho většinou českého, jest též oheň na deutschböhmské stř̌eše.“

67 Vgl. N. N.: Die Geistlichkeit und die Volkszählung. In: Aussiger Anzeiger, 24. 1. 1891.

68 N. N.: Štvanice na českého kněze. In: Česká stráž, 21. 6. 1890: „A přece jest známo, že kněží národnosti německé není v Čechách tolik, aby se těmto choutkám německým hovělo, kdyby se i chtělo.“ 
soll seiner Nationalität entsagen. “69 Der Verfasser sei „neugierig, wie weit dieser deutschböhmische Furor sich noch wagen “70 werde.

Die Forderung „Kauft nur bei Deutschen, unterstützt nur Deutsche“, die der Aussiger Anzeiger z. B. im Zusammenhang mit dem Trebnitzer Tierarzt propagierte, welcher sich bei der Volkszählung zur tschechischen Umgangssprache bekannte, hatte ihre tschechische Entsprechung: ,Svůj k svému' (Jeder zu den Seinen). Die tschechische Version der Parole wurde in der Zeitung Česká stráž zum einen ebenfalls im Kontext der Volkszählung von 1890 häufig wiederholt, zum anderen erschien sie auf den Zeitungsseiten oft als ein selbstständiger Aufruf im Anzeigenteil, groß- und fettgedruckt. Diese Forderung scheint sogar zu den ideellen Grundlagen der Zeitung Česká stráž gehört zu haben: Bereits in der Gründungsnummer dieses Blattes beteurte der Bürgermeister von Trebnitz Václav Pařík: „Wir müssen uns volkswirtschaftlich organisieren, materiell organisieren. Wir müssen uns bemühen, das Kapital in unseren Händen, in den tschechischen Händen zu akkumulieren und uns aus der Vorherrschaft des deutschen Kapitals zu befreien."71 Dies scheint den Lesern nichts anderes suggerieren zu wollen, als dass sie ihr Konsumverhalten gerade nach dem Kriterium der Nationalität richten. Neben den isolierten Aufrufen ,Svůj k svému“ äußerte sich die Zeitung Česká stráž gelegentlich auch explizit zu dieser Problematik: „Diesen Grundsatz der Vergeltung lernen wir von unseren lieben Landsleuten der anderen Nationalität. Unsere mährischen Brüder in Proßnitz [Prostějov] führen diesen Grundsatz im praktischen Leben konsequent durch [...]“.72

Ähnlich wie die Parole ,Svůj k svému“ erschien auf den Seiten der Zeitung Česká stráž ebenfalls gelegentlich der isolierte Aufruf „Gedenket der Volkszählung, die für den 31. Dezember bestimmt ist, und bereitet Euch vor!"73 Im Unterschied zum Aussiger Anzeiger, in welchem der erste Artikel zum Thema Volkszählung erst am 17. Dezember 1890 erschien, wurde die Volkszählung in der tschechischen Zeitung bereits in den Sommermonaten und im Herbst 1890 thematisiert. ${ }^{74}$ Der Aussiger Anzeiger hatte also nicht ganz Unrecht, als er im Nachhinein bemerkte: „Die tschechischen Blätter strotzten von den fetten Lettern der beweglichsten Aufrufe [...]“.75

69 N. N.: Štvanice na českého kněze. In: Česká stráž, 21. 6. 1890: „A tu má přinésti kněz původu českého obět, kterou v působišti ryze českém nepřináší ani nejpodřízenější sluha německého jazyka; má se totiž zříci své národnosti.“

70 N. N.: Štvanice na českého kněze. In: Česká stráž, 21. 6. 1890: „Jsme zvědavi, jak daleko deutschböhmský tento furor ještě se opováží [...]“.

71 Václav Pařík: [ohne Titel]. In: Česká stráž, 1. 1. 1890: „Musíme se organisovati národohospodářsky - hmotně. Musíme se snažiti kapitál v rukou svých, v rukou českých soustřediti a vymaniti se z područí kapitálu německého." Hervorhebung im Original gesperrt.

72 N. N.: Svůj k svému. In: Česká stráž, 23. 8. 1890: „Této zásadě odvety naučíme se dle všeho od našich milých krajanů národnosti druhé. Naši bratř̌i moravští v Prostějově provádějí totiž zásadu ,svůj k svému důsledně v praktickém životě, kupujíce potřeby své toliko od našinců, obchodníkủ českých.“

73 „Pamatujte a připravujte se na sčítání lidu na den 31. prosince 1890 určené!“ (Z. B. Česká stráž, 20. 9. 1890 u. a. m.)

74 Vgl. z. B. N. N.: Sčítání lidu. In: Česká stráž, 30. 8. 1890; N. N.: Germanisace na jihu. In: Česká stráž, 20. 9. 1890; N. N.: Sčítání lidu roku 1890. In: Česká stráž, 11. 10. 1890; N. N.: Sčítání lidu a němečtí nacionálové v Chebu. In: Česká stráž, 18. 10. 1890 u. a. m.

75 N. N: Der Krieg auf dem Zählbogen. In: Aussiger Anzeiger, 17. 1. 1891. 


\section{Schlussbetrachtung}

Als das dominante Argumentationsmuster des Aussiger Anzeigers kristallisiert sich das spezifische Verständnis des Ausdrucks ,Umgangssprache“ heraus. Es sei diejenige Sprache, die eine Person im alltäglichen Umgang am häufigsten verwendet. Diesem Verständnis zufolge sollte selbst ein tschechischer Muttersprachler, wenn er in einer überwiegend deutschsprachigen Umgebung lebt und arbeitet, als seine Umgangssprache Deutsch angeben. Die tschechischsprachige Zeitung Česká stráž wiederum setzt die Umgangssprache mit der Nationalität gleich. Darüber hinaus bemühen sich sowohl der Aussiger Anzeiger als auch die Zeitung Česká stráž um eine Polarisierung und strikte Trennung nach dem - vagen - Kriterium der Nationalität und argumentieren mit Zuordnungen ,deutsch', ,Deutsche“ (eventuell mit den ultimativen Bezeichnungen ,reindeutsch' oder ,kerndeutsch') resp. ,tschechisch` und ,Tscheche`, die jedoch zumeist durch keine oder nur vage Begründungen gestützt sind. Die diametral unterschiedlichen Angaben zu den Bewohnerzahlen der Gemeinde Trebnitz, die im Aussiger Anzeiger und in der Zeitung Česká stráž angeführt wurden, exemplifizieren, dass einige Redakteure für ihre kommunikativen Ziele nicht zuletzt angebliche statistische Daten instrumentalisieren wollten. Im Aussiger Anzeiger kommt als ein weiteres Argument die Warnung vor dem historischen Recht der Länder der böhmischen Krone hinzu, dessen Verwirklichung eine größere Autonomie der böhmischen Länder bedeutet hätte.

\section{Quellen- und Literaturverzeichnis}

Aussiger Anzeiger, 1890-1891

Česká stráž, 1890-1891

DIJK, Teun van (2008): Discourse and Power. Chippenham, Eastbourne.

HOENSCH, Jörg K. (1997): Geschichte Böhmens. Von der slavischen Landnahme bis zur Gegenwart. München.

KANN, Robert A. (1964): Das Nationalitätenproblem der Habsburgermonarchie. Geschichte und Ideengehalt der nationalen Bestrebungen vom Vormärz bis zur Auflösung des Reiches im Jahre 1918. 1. Bd. Graz, Köln.

NIEHR, Thomas (2014): Einführung in die linguistische Diskursanalyse. Darmstadt.

NIEHR, Thomas (2014): Einführung in die Politolinguistik. Göttingen.

PAVLÍČKOVÁ, Tereza (2013): Die Entwicklung des Nationalitätenkonflikts in der Znaimer deutschen Presse 1850-1938. Olomouc.

SPIEß, Constanze (2008): Linguistische Diskursanalyse als Mehrebenenanalyse - Ein Vorschlag zur mehrdimensionalen Beschreibung von Diskursen aus forschungspraktischer Perspektive. In: WARNKE, Ingo, SPITZMÜLLER, Jürgen (Hrsg.): Methoden der Diskurslinguistik. Sprachwissenschaftliche Zugänge zur transtextuellen Ebene. Berlin, New York, S. 237-259.

TEIBENBACHER, Peter, KRAMER, Diether, GÖDERLE, Wolfgang (2012): An Inventory of Austrian Census Materials, 1857-1910. Final Report. In: Mosaic Working Paper WP2012-007, Max Planck Institute for Demographic Research: December 2012. http://www.censusmosaic.org/sites/default/files/downloads/publications/mosaicWP/MOSAIC-WP-2012-007.pdf (17. 5. 2016). 
WARNKE, Ingo, SPITZMÜLLER, Jürgen (2008): Methoden und Methodologie der Diskurslinguistik - Grundlagen und Verfahren einer Sprachwissenschaft jenseits textueller Grenzen. In: WARNKE, Ingo, SPITZMÜLLER, Jürgen (Hrsg.): Methoden der Diskurslinguistik. Sprachwissenschaftliche Zugänge zur transtextuellen Ebene. Berlin, New York, S. 3-54.

WENGELER, Martin (2008): „Ausländer dürfen nicht Sündenböcke sein“ - Diskurslinguistische Methodik, präsentiert am Beispiel zweier Zeitungstexte. In: WARNKE, Ingo, SPITZMÜLLER, Jürgen (Hrsg.): Methoden der Diskurslinguistik. Sprachwissenschaftliche Zugänge zur transtextuellen Ebene. Berlin, New York, S. 207-236.

Mgr. Tereza Hrabcová, PhD. / Tereza.Hrabcova@ujep.cz

Univerzita Jana Evangelisty Purkyně v Ústí nad Labem, Filozofická fakulta, Katedra germanistiky, Pasteurova 13, 40096 Ústí nad Labem, CZ 